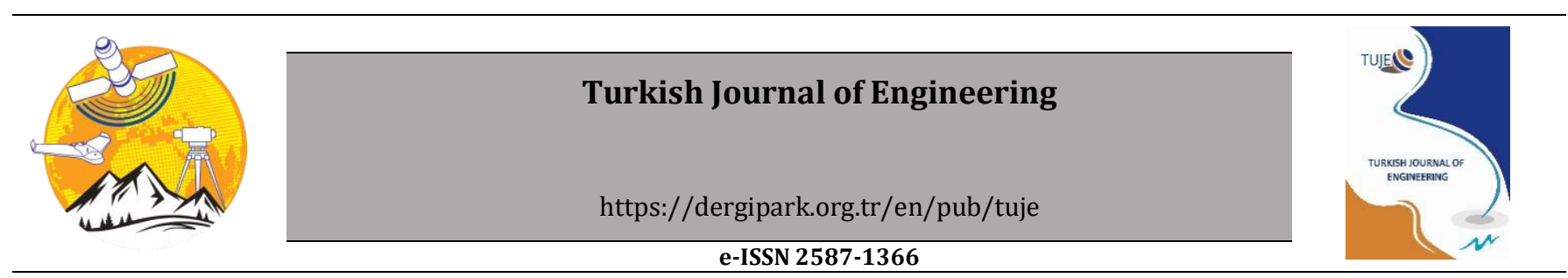

\title{
Retrieving the SNR metrics with different antenna configurations for GNSS-IR
}

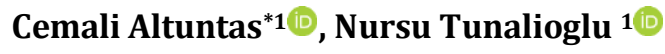 \\ ${ }_{1}^{1}$ Yildiz Technical University, Faculty of Civil Engineering, Department of Geomatics Engineering, Istanbul, Turkey
}

\author{
Keywords \\ Multipath \\ Signal-to-noise ratio (SNR) \\ GNSS Interferometric \\ Reflectometry (GNSS-IR) \\ Smartphone \\ Horizon-looking antenna
}

\begin{abstract}
Multipath, which is a major source of error for precise positioning, is the effect that occurs when Global Navigation Satellite Systems (GNSS) signals reach the receiver by reflecting from one or more surfaces. Reflected signals affect the signal-to-noise ratio (SNR) data provided by the receiver, indicating the signal strength. The structure of the antenna of the receiver and the direction in which it is oriented also change the strength of the received signal. In this study, the effect of antenna orientation and polarization on SNR data was demonstrated by using the method called GNSS-Interferometric Reflectometry (GNSS-IR), in terms of reflector height estimates. A geodetic GNSS receiver (CHC i50) and two different smartphones (Xiaomi Mi8 and Xiaomi Mi8 Lite) were used in the four-day experiments. The geodetic receiver was established as zenith-looking (ZL) in the first two days and as horizon-looking (HL) in the last two days. Smartphones were placed on the same mast with the HL receiver in the last two days. It was seen that it is more appropriate to use a $0^{\circ}-60^{\circ}$ satellite elevation angle range in the common use of all receivers' data. In the $30^{\circ}-60^{\circ}$ range where the ZL installation receives the multipath signals weakly, it has been found that the HL receiver and smartphones have reflector height estimation accuracies with values ranging from $1.9 \mathrm{~cm}$ to $2.5 \mathrm{~cm}$. In short, for different elevation angle ranges, accuracies below $2 \mathrm{~cm}$ could be obtained with each receiver. Thus, different antenna configurations may be used in GNSS-IR studies, depending on the characteristics of the study area and the surface feature to be determined.
\end{abstract}

\section{INTRODUCTION}

Global Navigation Satellite Systems (GNSS) enable to estimate accurate three-dimensional (3D) Cartesian coordinates of the points where the receivers are located to desired locations to be computed. As commonly known, the signals received from GNSS satellites are subjected to various error sources depend on the satellite error, receiver error, geophysical error, and atmospheric error, etc. If the GNSS signal arrives at the receiving antenna with more than one or more paths by reflecting off surfaces, multipath error on the signal occurs due to those reflections. The traveling time of the reflected signal is slightly delayed when compared with the direct signal, which results in inaccurate positioning calculations. Among the error sources of GNSS, multipath is one of the major and significant error, which referred to as unmodelled, should be eliminated from the observations when accurate positioning is required. To reduce this effect on observations, location selection of
GNSS station such as establishing away from the reflective surfaces, and antenna type selection such as suppressing the multipath are the basic requirements. However, multipath, which is an undesired error source for GNSS positioning, has become an effective tool to determine the reflective surface characteristics recently.

The methodology of the GNSS-Interferometric Reflectometry (GNSS-IR) was first introduced by MartinNeira (1993) to perform ocean altimetry for retrieving the height of the ocean over a reference ellipsoid. After that, in many studies related to the estimation of the snowpack (Larson et al. 2009; Ozeki \& Heki 2012; Gutmann et al. 2012; Chen et al. 2014; Jin et al. 2016; Tunalioglu et al., 2019), soil moisture (Larson et al. 2008; Larson et al., 2010; Roussel et al. 2016; Zhang et al. 2017; Han et al. 2020; Altuntas \& Tunalioglu, 2020a), sea-level tide (Anderson 2000; Xi et al. 2018), deformation monitoring (Yang et al. 2019), etc., the GNSS-IR methodology has been implemented successfully. 
In this study, as the multipath effect is dominant at low elevation angles and the antenna gain pattern is significant to suppress this, two different settlement configurations of the GNSS antenna are implemented to assess the SNR metrics estimated. To do that, CHC i50 geodetic GNSS receiver is installed into two configurations as horizontal-looking (HL) and zenithlooking (ZL). The main aim of changing the orientation of the receiver installation is to examine the satellite elevation angle ranges over the signal-to-noise ratio (SNR) data recorded. Moreover, at simultaneous observation duration, the SNR data of the low-cost single-frequency GNSS chipset embedded android smartphones (Xiaomi Mi8 \& Xiaomi Mi8 Lite) attaching at mast are recorded where the polarization of the smartphone is linear, which makes the observations more sensitive to multipath.

\section{MATERIALS AND METHOD}

\subsection{Study Area and Experimental Setup}

The study area is a football stadium in Yildiz Technical University Davutpasa Campus. Since the field has a wide, flat, and slightly rough surface and far from buildings, the interferences of a single ground-reflected and direct signals are considered. There is an open view of the sky at the point where the receiver is installed (Figure 1).

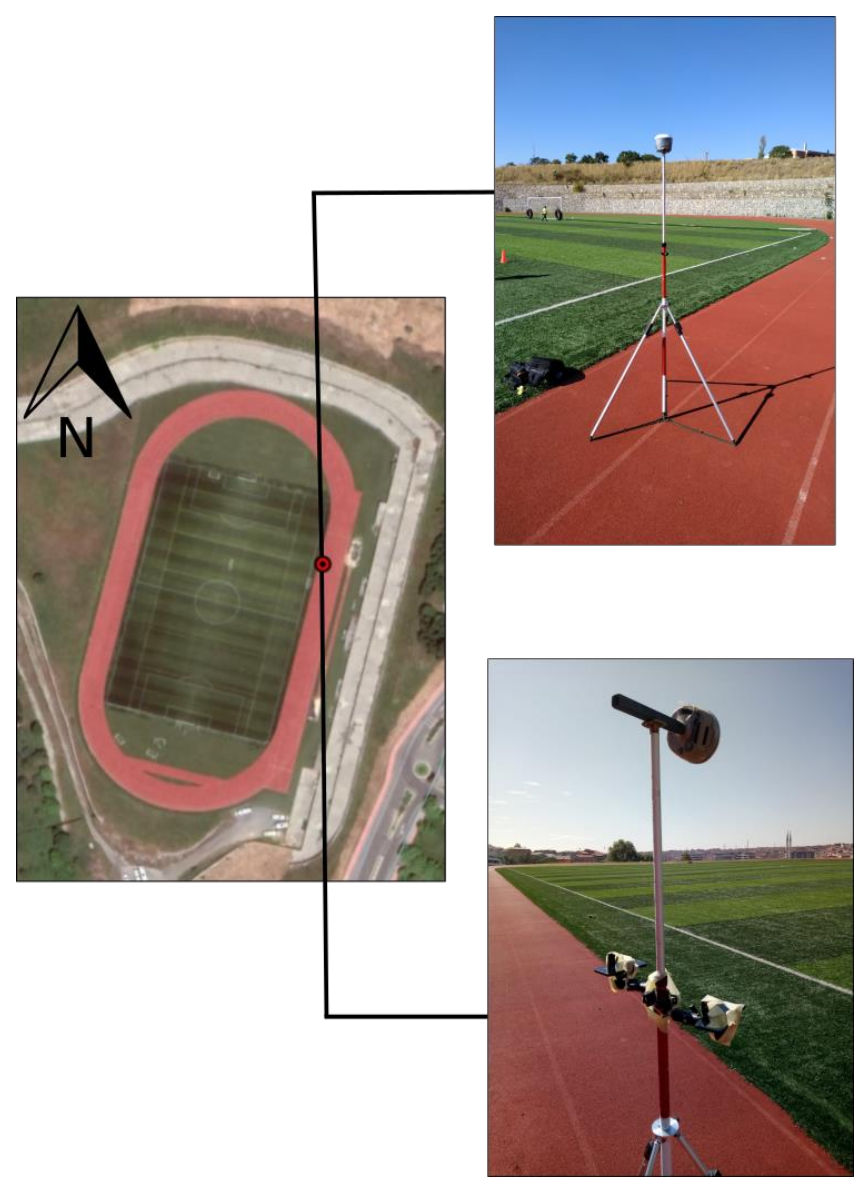

Figure 1. Study area and setup

Measurements were carried out on four different days. On 23-24 September 2020 (DoY: 267, 268), CHC i50 geodetic GNSS receiver was set up at the selected point, by orienting the antenna to the ZL. On 1-2 October 2020 (DoY: 275, 276), the geodetic GNSS receiver was oriented $\sim 250^{\circ}$ azimuth to the HL, while two smartphones named Xiaomi Mi8 (M8) and Xiaomi Mi8 Lite (M8L) are installed on the same mast. The vertical distances between the ground and the antenna phase centers of the receivers, i.e. reflector heights, are given in Table 1.

Table 1. In situ heights of the receivers

\begin{tabular}{llc}
\hline DoY & Receiver & h (m) \\
\hline 267,268 & CHC i50 (ZL) & 2.127 \\
275,276 & CHC i50 (HL) & 2.025 \\
275,276 & Xiaomi Mi8 & 1.320 \\
275,276 & Xiaomi Mi8 Lite & 1.380 \\
\hline
\end{tabular}

\subsection{GNSS Interferometric Reflectometry (GNSS-IR)}

The multipath effect is dominant at low satellite elevation angles due to the reflection surfaces surrounded at the GNSS receiver established. In a multipath-free environment, the direct signal transmitted from the satellite follows a direct path to reach the receiver, and the signal power equals to the amplitude of the direct signal $\left(A_{d}\right)$. If the signal reflects from a reflection surface, this results in attenuation of the amplitude of the reflected signal $\left(A_{m}\right)$, which is quite less than the amplitude of the direct signal referred to $A_{d} \gg$ $A_{m}$ (Bilich et al., 2008). Due to the reflection, the reflected signal follows longer path than the direct signal. The geometrical expression of this extra path denoted $\Delta \mathrm{s}$ occurred between direct and reflected signals can be seen in Figure 2, which can be formulated directly from the geometrical relationship as $2 h \sin \varepsilon$, where $h$ is the reflector height, $\varepsilon$ is the satellite elevation angle.

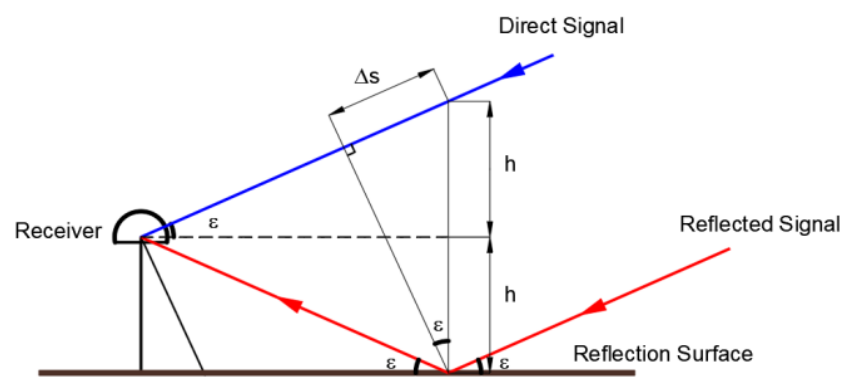

Figure 2. Geometry of multipath, $\Delta$ s refers to extra path, (Hoffmann-Wellenhof et al. 2007)

The direct and reflected signals interfere at the antenna phase center and are routinely recorded by the GNSS receiver simultaneously. The multipath effect causes oscillations on GNSS observations (Jin et al., 2016). The signal strength denoted as $S$ observable referred to the carrier-to-noise-density ratio (CNR or $\mathrm{C} / \mathrm{NO}$ ), which is the ratio of signal power to the noise power spectral density (Larson \& Nievinski, 2013). To assess the quality of the signal where the signal characteristics can be retrieved, the SNR (or S/N), which is related to $\mathrm{C} / \mathrm{NO}$ with noise bandwidth (assuming $1-\mathrm{Hz}$ ) is used after converting the units from the logarithmic scale (in a unit of decibel) to linear scale (in a unit of watts per watt or volts per volt if the square root is taken) (Larson \& Nievinski, 2013). If a single-reflected signal and direct signal recorded at the receiver are considered, 
the SNR value can be expressed as Equation 1 (Bilich et al., 2008),

$$
S N R^{2} \equiv A_{d}{ }^{2}+A_{m}{ }^{2}+2 A_{d} A_{m} \cos \Delta \varphi
$$

where $A_{d}$ and $A_{m}$ are the amplitude of direct and reflected signals in units of volts/volts, respectively; $\Delta \varphi$ is the multipath relative phase in radians, which is a function of satellite geometry.

In SNR observables, the contribution of the direct signal is not required for estimation of the SNR metrics, which are reflector height, phase, and amplitude of multipath, and should be removed from the SNR series for each satellite tracks. Since, as mentioned $A_{d} \gg A_{m}$, and $A_{d}$ doesn't contain reflection properties, the loworder polynomial fitting can be used to remove the contribution of direct signal from SNR observation series. Then, after removing the SNR trend, the detrended SNR denoted $d S N R$, which is multipath pattern can be expressed as given in Equation 2,

$d S N R=A \cos \left(\frac{4 \pi h}{\lambda} \sin \varepsilon+\phi\right)$

where $\lambda$ is the wavelength of the GNSS signal in meters, $\phi$ is the multipath phase in radians.

Here, the frequency of the multipath modulation (f) that is related to the $h$, can be written as $f=2 h / \lambda$, constant of a function of $\sin \varepsilon$. Once the dominant frequency of $d S N R$ is estimated, then it can be converted to the $h$ by using the mentioned equation.

\subsection{Data and Analysis}

Signal acquisition capabilities of the smartphones and the geodetic receivers are different from each other. The signals collected by the geodetic receiver and smartphones used in this study are given in Table 2 .

Table 2. SNR types of collected signals

\begin{tabular}{lll}
\hline Receiver & Satellite system & SNR signals \\
\hline CHC i50 & GPS & S1C, S2D, S5Q \\
& GLONASS & S1C, S2P \\
& Galileo & S1X, S5X, S7X \\
& BeiDou & S1I, S6I, S7I \\
\hline Xiaomi Mi8 & GPS & S1C, S5Q \\
& GLONASS & S1C \\
& Galileo & S1C, S5Q \\
& BeiDou & S2I \\
\hline Xiaomi Mi8 Lite & GPS & S1C \\
& GLONASS & S1C \\
& Galileo & S1C \\
& BeiDou & S2I \\
\hline
\end{tabular}

Considering the observation types, it is seen that a sufficient number of common observations are only in the SNR data for the signals collected at the L1 frequency. Therefore, only the SNR data of the L1 frequency signals (S1C, S1X, S1I) from all satellite systems were evaluated.

The observation period for a day is set to a minimum of 5 hours. The data sampling interval of the receivers was set to 1 second. The observations made with smartphones were recorded using the android application named Geo++ RINEX Logger.

Since some of the area surrounding the stadium is much higher than the stadium ground, the line of sight between the receiver-satellite is blocked in some azimuth ranges $\left(0^{\circ}-200^{\circ}\right.$ and $\left.300^{\circ}-360^{\circ}\right)$ for low satellite elevation angles. Therefore, the data from the $200^{\circ}-300^{\circ}$ azimuth range were considered (Figure 3). Satellite elevation angle limits are not taken as fixed and it is aimed to evaluate the results obtained for different intervals: 10-degree intervals between $0^{\circ}-60^{\circ}$, incremental intervals between $0^{\circ}-60^{\circ}$ and incremental intervals between $30^{\circ}-60^{\circ}$.

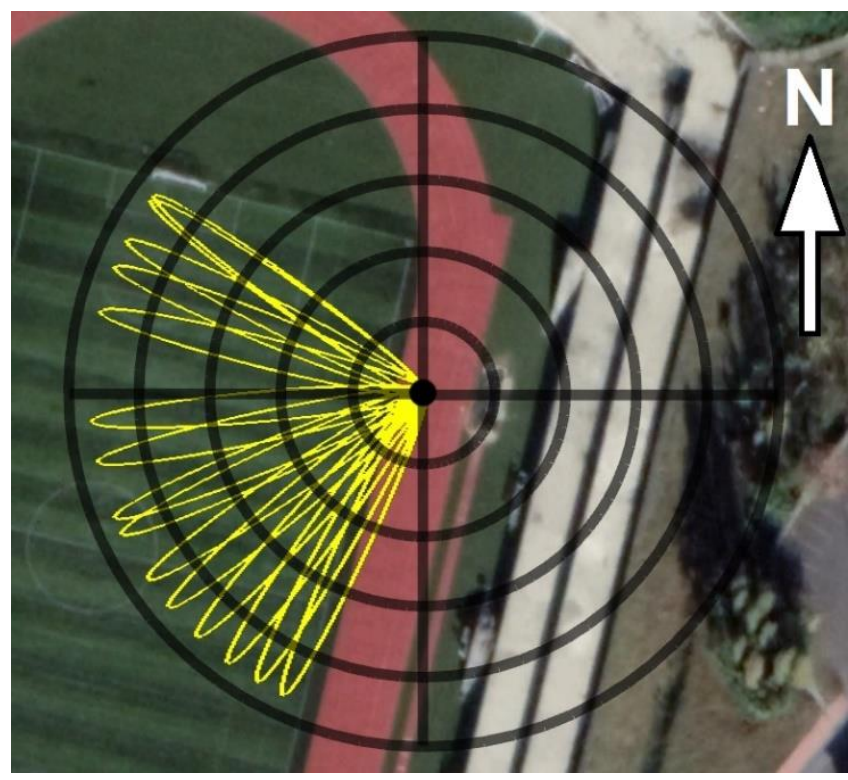

Figure 3. First Fresnel zones for $2 \mathrm{~m}$ antenna height and $5^{\circ}$ elevation angle in the $200^{\circ}-300^{\circ}$ azimuth range

SNR data were grouped as ascending and descending according to satellite transitions. A quadratic polynomial was used to remove the SNR trend. The dominant frequencies of the detrended SNR $(d S N R)$ signals were estimated using Lomb-Scargle Periodogram (LSP), which is a commonly used method to determine the periodicity of irregularly sampled data. The Median Absolute Deviation (MAD) method was used to detect the outliers (Leroy \& Rousseeuw 1987). 1MAD was selected as the test value depending on the results obtained by Altuntas \& Tunalioglu (2020b).

\section{RESULTS AND DISCUSSIONS}

The polarization of the antennas of geodetic GNSS receivers and smartphones are different. Geodetic receivers have circularly polarized antennas while smartphones have linearly polarized. In addition, the antenna gain patterns of geodetic receivers are designed to receive signals from low satellite elevation angles with minimum gain to reduce the contribution of affected signals (e.g. multipath signals). Due to differences in polarization and antenna gain pattern, smartphones are more sensitive to reflected signals than geodetic receivers. However, this statement is true when the geodetic receiver is set up in the classical way (i.e. oriented to the zenith direction). If the receiver is 
oriented so that its antenna is looking in a different direction than the zenith, the antenna gain pattern rotates with the antenna, and maximum gain is achieved in the direction that the antenna is looking.

When the receiver is oriented towards the horizon instead of the zenith, the maximum gain is obtained for a signal coming from $0^{\circ}$, not $90^{\circ}$. Considering that the multipath signals are more intense at low elevation angles, it can be expected that directing the receiver to the horizon (HL) will result in stronger reception of reflected signals.

Two common observation samples for $d S N R$ signals obtained from data collected by ZL receiver, HL receiver and smartphones are plotted in Figure 4. It is seen that the highest $d S N R$ amplitude is obtained with the HL receiver and the lowest amplitude is obtained with the ZL receiver. However, there is a critical issue that needs to be underlined here: orienting the receiver's antenna to a direction where the reflection signals are intense not only increases the $d S N R$ amplitude but also increases the noise of the $d S N R$ signal. Therefore, directly comparing the amplitudes of different receivers may cause misinterpretations. Instead, if each data set is normalized within itself and the normalized amplitudes are compared with each other, the results can be more unbiased and interpreted more accurately. Normalized $d S N R$ values can be obtained using the following equation:

$\operatorname{norm}\left(d S N R_{i}\right)=\frac{d S N R_{i}-\min (d S N R)}{\max (d S N R)-\min (d S N R)}$
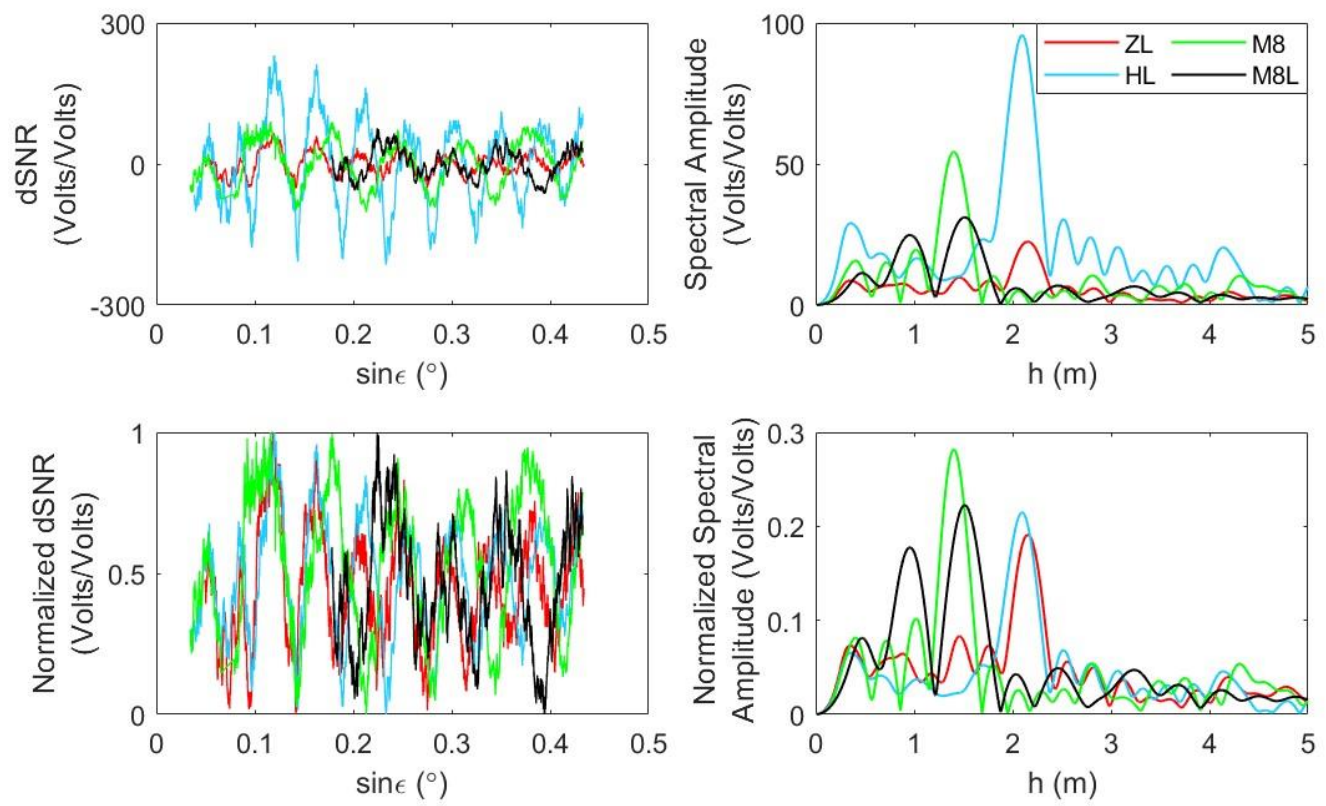

(a)
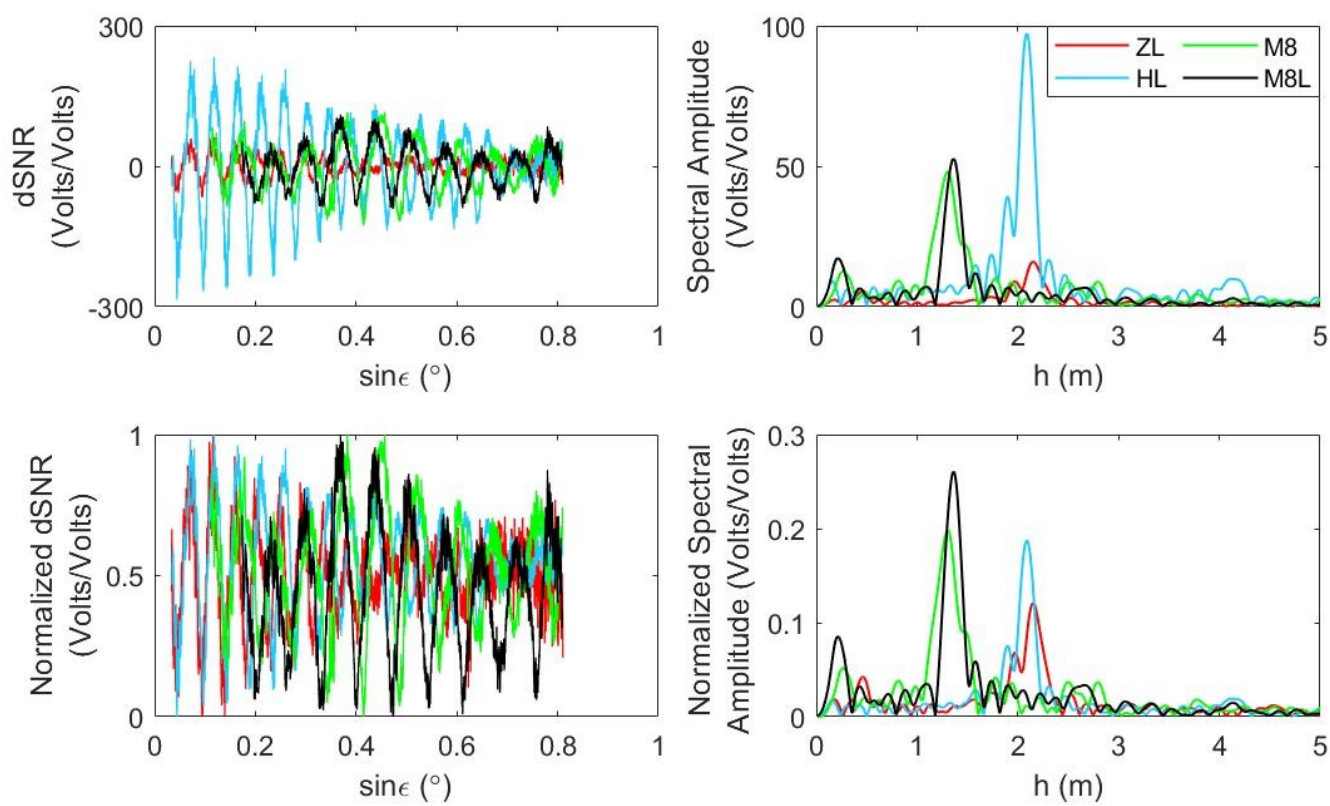

(b)

Figure 4. $d S N R$ and LSP plots with their normalized versions for (a) G01 Ascending data (b) G03 Ascending data 
Normalized $d S N R$ and their LSPs are given in Figure 4. In the data of the G01 satellite, it seems the strongest peak in spectral amplitudes was obtained with the HL receiver and the weakest peak with the ZL receiver. The ratio between these two values is $\sim 5$. However, considering the normalized spectral amplitudes, it is seen that the highest value was obtained with M8, and the values of HL and ZL receivers are close to each other. It can be seen that the situation is the same for the data of the G03 satellite. Therefore, it is more appropriate to consider normalized $d S N R$ rather than $d S N R$ when comparing different structured receivers. Accordingly, for the $0^{\circ}-60^{\circ}$ satellite elevation angle range, the lowest normalized spectral amplitude value is obtained with ZL receiver, while the values of HL receiver and smartphones are close to each other.

The reflector height estimations and root mean square errors (RMSEs) obtained when 10-degree satellite elevation angle intervals are selected, are given in Table 3. The lowest RMSE values were found as $4 \mathrm{~cm}$ in $10^{\circ}-20^{\circ}$ range for $Z \mathrm{~L}$ receiver, $2 \mathrm{~cm}$ in $0^{\circ}-10^{\circ}$ range for $\mathrm{HL}$ receiver, $5.9 \mathrm{~cm}$ in $0^{\circ}-10^{\circ}$ range for $\mathrm{M} 8$, and $4.1 \mathrm{~cm}$ in $40^{\circ}-50^{\circ}$ range for $\mathrm{M} 8 \mathrm{~L}$.

The results of the incremental ranges within the $0^{\circ}$ $60^{\circ}$ interval are given in Table 4 . The lowest RMSE values were found as $0.9 \mathrm{~cm}$ in $0^{\circ}-20^{\circ}$ range for ZL receiver, 2 $\mathrm{cm}$ in $0^{\circ}-10^{\circ}$ range for $\mathrm{HL}$ receiver, $3.3 \mathrm{~cm}$ in $0^{\circ}-60^{\circ}$ range for $\mathrm{M} 8$, and $1.9 \mathrm{~cm}$ in $0^{\circ}-30^{\circ}, 0^{\circ}-50^{\circ}$, and $0^{\circ}-60^{\circ}$ ranges for M8L.

The results of the incremental ranges within the $30^{\circ}$ $60^{\circ}$ interval are given in Table 5 . The lowest RMSE values were found as $9.3 \mathrm{~cm}$ for ZL receiver, $2.5 \mathrm{~cm}$ for $\mathrm{HL}$ receiver, $1.9 \mathrm{~cm}$ for $\mathrm{M} 8$, and $2.3 \mathrm{~cm}$ for M8L in $30^{\circ}-60^{\circ}$ range. Considering the values in these three tables, it is seen that the lowest RMSE is obtained when $0^{\circ}-60^{\circ}$ range is used. Therefore, it can be used as a common range for all of these receivers.

Table 3. Reflector height estimations and RMSEs for 10-degree intervals (in meters)

\begin{tabular}{cccrr}
\hline Range & CHC i50 (ZL) & CHC i50 (HL) & Xiaomi Mi8 & Xiaomi Mi8 Lite \\
\hline $0^{\circ}-10^{\circ}$ & $2.085 \pm 0.048$ & $2.028 \pm 0.020$ & $1.348 \pm 0.059$ & NO DATA \\
$10^{\circ}-20^{\circ}$ & $2.158 \pm 0.040$ & $2.114 \pm 0.090$ & $1.372 \pm 0.104$ & $1.435 \pm 0.143$ \\
$20^{\circ}-30^{\circ}$ & $2.123 \pm 0.110$ & $2.036 \pm 0.061$ & $1.279 \pm 0.069$ & $1.372 \pm 0.099$ \\
$30^{\circ}-40^{\circ}$ & $2.138 \pm 0.162$ & $2.032 \pm 0.031$ & $1.261 \pm 0.069$ & $1.321 \pm 0.067$ \\
$40^{\circ}-50^{\circ}$ & $2.030 \pm 0.274$ & $2.002 \pm 0.094$ & $1.235 \pm 0.120$ & $1.377 \pm 0.041$ \\
$50^{\circ}-60^{\circ}$ & $2.214 \pm 0.153$ & $2.035 \pm 0.092$ & $1.390 \pm 0.120$ & $1.591 \pm 0.282$ \\
\hline
\end{tabular}

Table 4. Reflector height estimations and RMSEs for incremental intervals between $0^{\circ}-60^{\circ}$ (in meters)

\begin{tabular}{rrrrr}
\hline Range & CHC i50 (ZL) & CHC i50 (HL) & Xiaomi Mi8 & Xiaomi Mi8 Lite \\
\hline $0^{\circ}-10^{\circ}$ & $2.085 \pm 0.048$ & $2.028 \pm 0.020$ & $1.347 \pm 0.059$ & NO DATA \\
$0^{\circ}-20^{\circ}$ & $2.128 \pm 0.009$ & $2.059 \pm 0.036$ & $1.360 \pm 0.064$ & $1.441 \pm 0.143$ \\
$0^{\circ}-30^{\circ}$ & $2.152 \pm 0.027$ & $2.060 \pm 0.043$ & $1.353 \pm 0.060$ & $1.374 \pm 0.019$ \\
$0^{\circ}-40^{\circ}$ & $2.136 \pm 0.027$ & $2.052 \pm 0.042$ & $1.343 \pm 0.053$ & $1.366 \pm 0.024$ \\
$0^{\circ}-50^{\circ}$ & $2.127 \pm 0.034$ & $2.053 \pm 0.041$ & $1.334 \pm 0.042$ & $1.371 \pm 0.019$ \\
$0^{\circ}-60^{\circ}$ & $2.124 \pm 0.034$ & $2.049 \pm 0.040$ & $1.321 \pm 0.033$ & $1.374 \pm 0.019$ \\
\hline
\end{tabular}

Table 5. Reflector height estimations and RMSEs for incremental intervals between $30^{\circ}-60^{\circ}$ (in meters)

\begin{tabular}{cccrr}
\hline Range & CHC i50 (ZL) & CHC i50 (HL) & Xiaomi Mi8 & Xiaomi Mi8 Lite \\
\hline $30^{\circ}-40^{\circ}$ & $2.138 \pm 0.162$ & $2.032 \pm 0.031$ & $1.261 \pm 0.069$ & $1.321 \pm 0.067$ \\
$30^{\circ}-50^{\circ}$ & $2.068 \pm 0.129$ & $2.028 \pm 0.033$ & $1.293 \pm 0.044$ & $1.372 \pm 0.031$ \\
$30^{\circ}-60^{\circ}$ & $2.078 \pm 0.093$ & $2.015 \pm 0.025$ & $1.306 \pm 0.019$ & $1.383 \pm 0.023$ \\
\hline
\end{tabular}

When all the results are reviewed, it can be said that an accuracy of $0.9 \mathrm{~cm}$ for $0^{\circ}-20^{\circ}$ range, of $2 \mathrm{~cm}$ for $0^{\circ}-10^{\circ}$ range, of $1.9 \mathrm{~cm}$ for $30^{\circ}-60^{\circ}$ range, and of $1.9 \mathrm{~cm}$ for $0^{\circ}$ $30^{\circ}, 0^{\circ}-50^{\circ}$ and $0^{\circ}-60^{\circ}$ ranges can be obtained from $\mathrm{CHC}$ i50 (ZL), CHC i50 (HL), Xiaomi Mi8 and Xiaomi Mi8 Lite, respectively. According to these results, for altering elevation angle ranges, an estimation accuracy of $\leq 2 \mathrm{~cm}$ could be obtained with each receiver.

Reflector height estimations and RMSEs are shown in Figure 5 with bar graphs and error bars. In order to ensure easy reading of the graphic, the vertical scale in Figure 5(a) was arranged as $1 \mathrm{~m}$, while it is $40 \mathrm{~cm}$ in Figure 5(b) and Figure 5(c). The red lines show the insitu measurements of reflector heights. According to the figure, the error bars in all of the estimates include the insitu height value.
In Figure 5(a), it is seen that the HL receiver's reflector height estimates are particularly accurate. In the ZL receiver, it is seen that the error bars widen in the data after $30^{\circ}$ and the difference between the estimations and the in-situ height is increased.

According to the Figure 5(b), it can be said that increasing the range in the ZL receiver improves the estimates, but the RMSE value increases with the inclusion of data from angles greater than $30^{\circ}$. Moreover, the $0.9 \mathrm{~cm}$ value obtained for 22 estimations made with the data from the $0^{\circ}-20^{\circ}$ range is the smallest RMSE value. For the HL receiver, the closest estimates to the insitu height were obtained with the data from the $0^{\circ}-10^{\circ}$ range. Increasing the angle range did not have a significant effect when angles greater than $20^{\circ}$ were included. The RMSE was around $\sim 4 \mathrm{~cm}$. In the data of Mi8 
and Mi8 Lite, the results improved as the angle range increased.

As seen in Figure 5(c), the worst results were obtained with the ZL receiver as expected. This is because the ZL receiver has the poor capability to collect reflected signals for the $30^{\circ}-60^{\circ}$ range. Estimations close
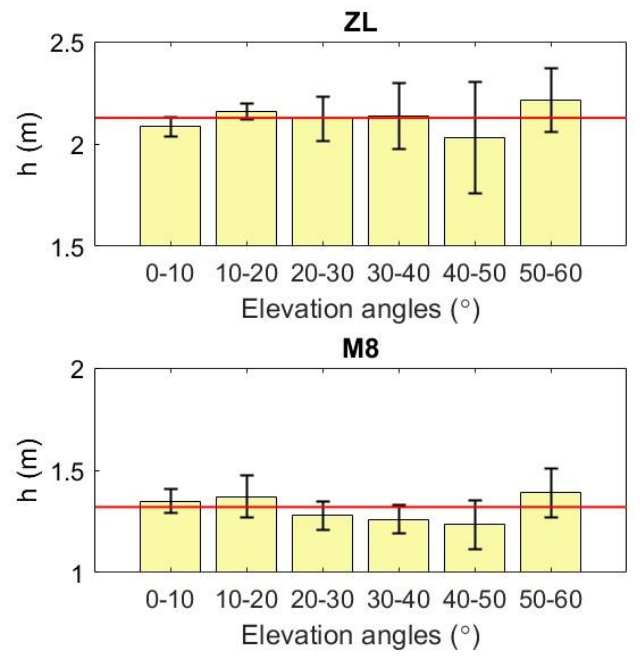

(a)

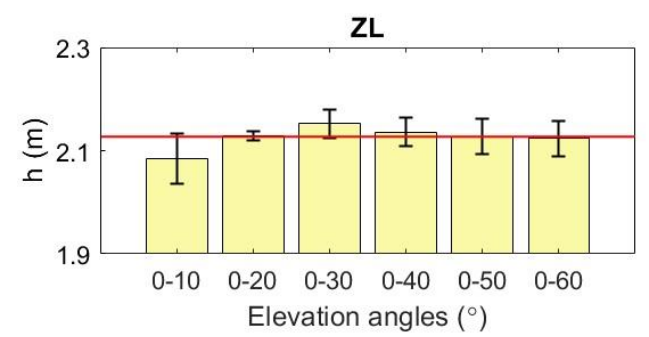

M8

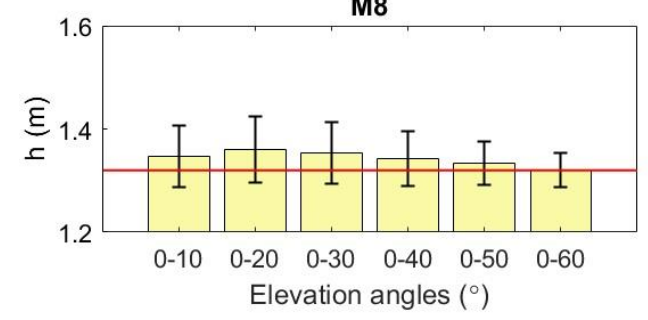

(b)
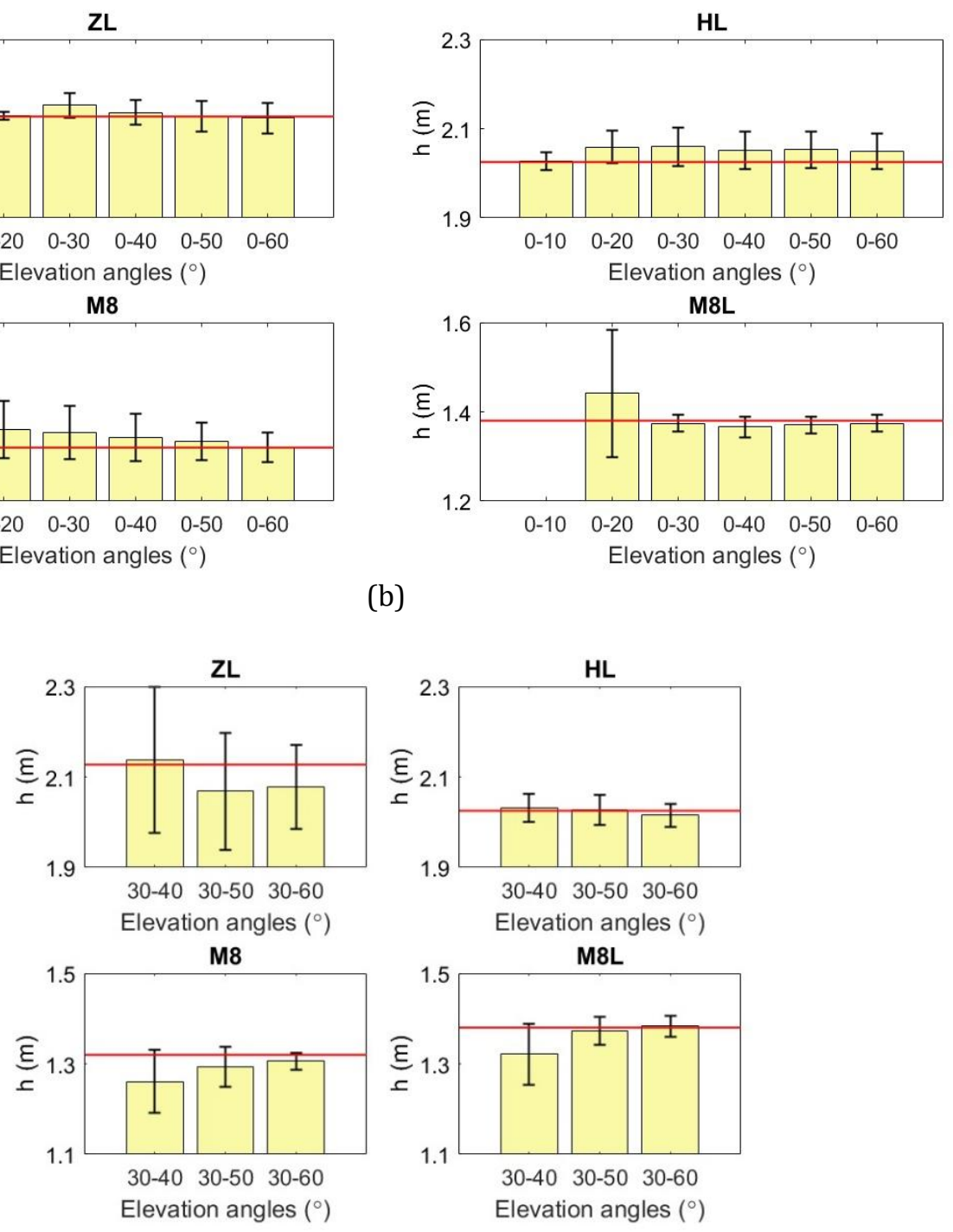

(c)

Figure 5. Reflector height estimations with error bars for (a) 10-degree intervals (b) incremental intervals between $0^{\circ}$ $60^{\circ}$ (c) incremental intervals between $30^{\circ}-60^{\circ}$

to the in-situ height with the HL receiver were obtained with an RMSE value of $\sim 3 \mathrm{~cm}$. Increasing the angle range has clearly improved the smartphone estimations. Considering the data from the $30^{\circ}-60^{\circ}$ range, the RMSE values of $1.9 \mathrm{~cm}$ with Mi8 and $2.3 \mathrm{~cm}$ with Mi8 Lite were found.
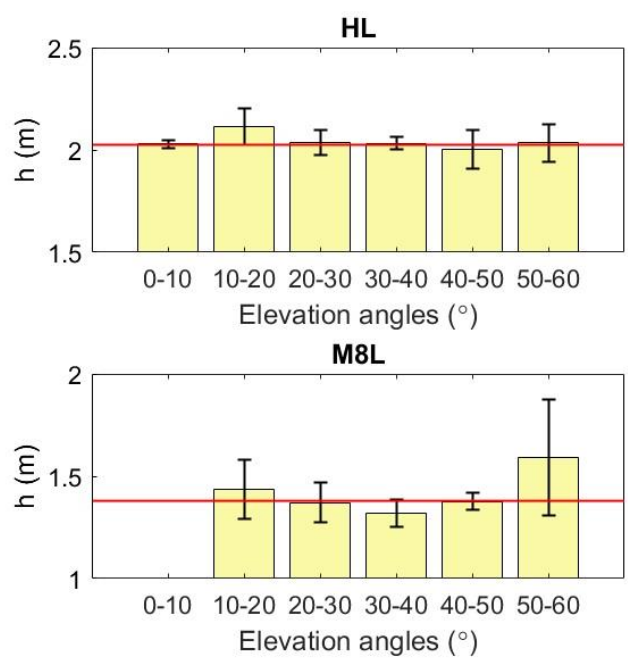
RMSE values are shown comparatively in Figure 6. Considering 10-degree intervals, it is seen that RMSEs were greater than $10 \mathrm{~cm}$ at angles greater than $20^{\circ}$ for the ZL receiver. For Mi8 Lite, it is seen that the RMSEs decrease as the elevation angle is increased. However, the result is bad in the $50^{\circ}-60^{\circ}$ range. In the incremental intervals between $0^{\circ}-60^{\circ}$, it is seen that the average RMSE value from $0^{\circ}-30^{\circ}$ was below $5 \mathrm{~cm}$. It can also be said that the minimum RMSE can be obtained at $0^{\circ}-60^{\circ}$ when all receivers are used. In the incremental intervals between $30^{\circ}-60^{\circ}$, it is seen that the ZL receiver gives $\sim 3$ times worse results than other receivers. The data in this range is better provided by HL receiver and smartphones (with an average RMSE value of $2.2 \mathrm{~cm}$ ). Here, it can be said that increasing the range improves the results in general, even if the lover limit is $0^{\circ}$ or $30^{\circ}$.

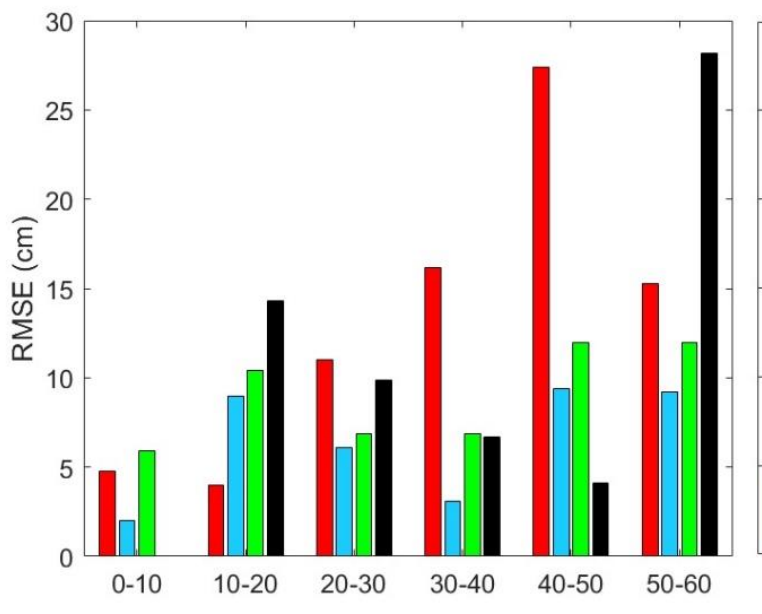

Figure 6. Comparative plot of RMSEs of estimations

\section{CONCLUSION}

SNR metrics associated with the estimation of environmental characteristics, where the GNSS signals reflect around the receiver installed can be determined by following the GNSS-IR method routinely in nowadays. The method has been implemented on ecosystem facilities related with snowpack determination, sea-level estimation, vegetation monitoring, soil moisture estimation etc., which are significant components for forecasting the climatology studies.

In GNSS-IR methodology, to record a sufficient number of satellite tracks where the strong reflections are included is required. Moreover, low elevation angles, routinely $5^{\circ}-25^{\circ}$ are capable of involving multipath effect than higher elevation angles in case of ZL installation. However, changing the orientation of the receiver in the manner of antenna gain pattern or using a linear polarized antenna, which is more sensitive to multipath than circular polarization may affect the estimations of SNR metrics.

In this study, a geodetic receiver with two different orientation configurations as $\mathrm{HL}$ and $\mathrm{ZL}$, and two different low-cost single-frequency GNSS chipset embedded android smartphones (M8 and M8L) with linear polarization were used for accuracy assessment of estimations. The data collected were investigated for a wide range of satellite elevation angles as $0^{\circ}-60^{\circ}$. The range is also divided into subsections with $10^{\circ}$ interval to examine the influence of the length of the SNR series on estimations. Normalized $d S N R$ values are used for comparative issues. In-situ measurements of reflector heights are used to compute the RMSE values. According to the results, the accurate results for HL installation of the geodetic receiver are obtained for $0^{\circ}-10^{\circ}$ and $30^{\circ}-60^{\circ}$ satellite elevation angles. When the configuration of the geodetic receiver was turned to ZL, the more well-agreed results with in-situ measurements were provided at $0^{\circ}$ $20^{\circ}$ and $0^{\circ}-30^{\circ}$ satellite elevation angle ranges. For the low-cost single-frequency GNSS chipset embedded android smartphones attached to the mast, the common elevation angle range agreed well with in-situ measurements is provided at $30^{\circ}-60^{\circ}$ for accuracy assessment. In particular, it can be concluded from the findings, for the studies related to the GNSS-IR method, the configuration of the receivers should be considered depending on the study content. Additionally, the contribution of the use of different configurations may be tested for further studies such as deformation monitoring, soil moisture estimation, and so on.

\section{Author contributions}

Cemali Altuntas: Conceptualization, Methodology, Software, Writing-Original draft preparation, Visualization Nursu Tunalioglu: Conceptualization, Methodology, Writing-Original draft preparation, Writing-Reviewing and editing, Supervision

\section{Conflict of interest}

The authors declare that they have no known relevant competing financial or non-financial interests that could have appeared to influence the work reported in this paper.

\section{REFERENCES}

Altuntas C \& Tunalioglu N (2020a). Estimation performance of soil moisture with GPS-IR method. Sigma Journal of Engineering and Natural Sciences, 38(4), 2217-2230.

Altuntas C \& Tunalioglu N (2020b). An experimental study on the effect of antenna orientation on GNSS-IR. $1^{\text {st }}$ Intercontinental Geoinformation Days, pp. 244-247. 
Anderson K D (2000). Determination of water level and tides using interferometric observations of GPS signals. Journal of Atmospheric and Oceanic Technology. 17(8), 1118-1127, doi:10.1175/15200426(2000)017<1118:DOWLAT>2.0.CO;2.

Bilich A, Larson K M \& Axelrad P (2008). Modeling GPS phase multipath with SNR: Case study from the Salar de Uyuni, Boliva, Journal of Geophysical Research, 113, B04401, doi:10.1029/2007JB005194.

Chen Q, Won D \& Akos D M (2014). Snow depth sensing using the GPS L2C signal with a dipole antenna. EURASIP Journal on Advances in Signal Processing, 1(106). doi:10.1186/1687-6180-2014-106.

Gutmann E D, Larson K M, Williams M W, Nievinski F G \& Zavorotny V (2012). Snow measurement by GPS interferometric reflectometry: an evaluation at Niwot Ridge, Colorado. Hydrological Processes, 26(19), 2951-2961, doi:10.1002/hyp.8329.

Han M, Zhu Y, Yang D, Chang Q, Hong X \& Song S (2020). Soil moisture monitoring using GNSS interference signal: proposing a signal reconstruction method. Remote Sensing Letters, 11(4), 373-382. DOI: 10.1080/2150704X.2020.1718235.

Hofmann-Wellenhof B, Lichtenegger H \& Wasle E (2007). GNSS-global navigation satellite systems: GPS, GLONASS, Galileo, and more. Springer Science \& Business Media.

Jin S, Qian X \& Kutoglu H (2016). Snow depth variations estimated from GPS-Reflectometry: A case study in Alaska from L2P SNR data. Remote sensing, 8(1), 63. doi:10.3390/rs8010063.

Larson K M, Nievinski F G (2013). GPS snow sensing: results from the EarthScope Plate Boundary Observatory, GPS Solut (2013) 17:41-52, DOI 10.1007/s10291-012-0259-7.

Larson K M, Small E E, Gutmann E D, Bilich A L, Braun J J \& Zavorotny V U (2008). Use of GPS receivers as a soil moisture network for water cycle studies. Geophysical Research Letters, Vol. 35, L24405, doi:10.1029/2008GL036013.

Larson K M, Gutmann E D, Zavorotny V U, Braun J J, Williams M W \& Nievinski F G (2009). Can we measure snow depth with GPS receivers?. Geophysical Research Letters, 36(17), doi:10.1029/2009GL039430.
Larson K M, Braun J J, Small E E, Zavorotny V U, Gutmann E D \& Bilich A L (2010). GPS Multipath and Its Relation to Near-Surface Soil Moisture Content. IEEE Journal of Selected Topics in Applied Earth Observations and Remote Sensing, 3(1), 91-99. doi: 10.1109/JSTARS.2009.2033612.

Leroy A M \& Rousseeuw P J (1987). Robust regression and outlier detection. Wiley Series in Probability and Mathematical Statistics, New York.

Martin-Neira M (1993). A passive reflectometry and interferometry system (PARIS): Application to ocean altimetry. ESA Journal, 17(4), 331-355.

Ozeki M \& Heki K (2012). GPS snow depth meter with geometry-free linear combinations of carrier phases. Journal of Geodesy, 86(3), 209-219, doi:10.1007/s00190-011-0511-x.

Roussel N, Frappart F, Ramillien G, Darrozes J, Baup F, Lestarquit L \& Ha M C (2016). Detection of Soil Moisture Variations Using GPS and GLONASS SNR Data for Elevation Angles Ranging From $2^{\circ}$ to $70^{\circ}$. IEEE Journal of Selected Topics in Applied Earth Observations and Remote Sensing, 9 (10), 4781.

Tunalioglu N, Dogan A H, Durdag U M (2019). GPS sinyal gürültü oranı verileri ile kar kalınlığının belirlenmesi. HKMOJJD, 6(1) 1-9. Doi: 10.9733/JGG.2019R00601001.T.

Xi R, Zhou X, Jiang W \& Chen Q (2018). Simultaneous estimation of dam displacements and reservoir level variation from GPS measurements. Measurement, 122, 247-256. doi:10.1016/j.measurement.2018.03.036.

Yang Y, Zheng Y, Yu W, Chen W \& Weng D (2019). Deformation monitoring using GNSS-R technology. Advances in Space Research 63 (2019) 3303-3314.

Zhang S, Roussel N, Boniface K, Ha M C, Frappart F, Darrozes J, Baup F \& Calvet J C (2017). Use of reflected GNSS SNR data to retrieve either soil moisture or vegetation height from a wheat crop. Hydrol. Earth Syst. Sci., 21, 4767-4784, https://doi.org/10.5194/hess-21-4767-2017. 